\title{
A eficácia do tratamento fisioterapêutico da incontinência urinária masculina após prostatectomia
}

\author{
Efficacy of Physical Therapy for Male Urinary Incontinence Following Prostate \\ Removal
}

Livia Marie Kubagawa', José Renato Ferreira Pellegrini', Vanessa Pereira de Lima², Adriana Luciana Moreno ${ }^{3}$

\section{Resumo}

O câncer de próstata é a malignidade mais comumente detectada em homens em países industrializados e a prostatectomia radical é o método de tratamento mais eficaz para esse tipo de câncer. No entanto, essa operação causa algumas complicações, entre elas, a incontinência urinária. Este trabalho é uma revisão bibliográfica sobre a eficácia da atuação fisioterapêutica no tratamento da incontinência urinária em prostatectomizados. O mesmo aborda o câncer de próstata, bem como as suas formas de tratamento com destaque para a prostatectomia e suas possíveis complicações, enfatizando a incontinência urinária, sua fisiopatologia e o seu respectivo tratamento fisioterapêutico através da eletroestimulação, biofeedback, treinamento comportamental e exercícios para o assoalho pélvico. Todas as abordagens mencionadas foram comprovadas, pelos autores consultados, como eficazes para a diminuição dos sintomas urinários e obtenção mais rápida da continência urinária.

Palavras-chave: Prostatectomia, Incontinência urinária, Fisioterapia.

\section{Abstract}

Prostate cancer is the most frequently detected malignancy in men in industrialized countries, and radical prostatectomy is the most effective method for treating this cancer. However, the surgery can lead to some complications, including urinary incontinence. The current study reviews the efficacy of physical therapy in the treatment of postprostatectomy urinary incontinence. The study focuses on prostate cancer and its treatment modalities, specifically prostatectomy and possible complications such as urinary incontinence, pathophysiological aspects, and the respective physical therapy approaches: electric stimulation, biofeedback, behavioral training, and pelvic floor exercises. All the authors consulted demonstrated that the above-mentioned approaches can improve urinary symptoms and achieve earlier urinary continence.

Key words: Prostatectomy, Urinary incontinence, Physical therapy.

\footnotetext{
${ }^{1}$ Fisioterapeuta

${ }^{2}$ Fisioterapeuta Mestranda em Ciências Médicas pela Unifesp e docente do Centro Universitário São Camilo.

${ }^{3}$ Fisioterapeuta Doutoranda em Ciências da Saúde da Disciplina de Ginecologia pela Unifesp e docente do Centro Universitário São Camilo. Trabalho realizado no Centro Universitário São Camilo.

Endereço para correspondência: Lívia Marie Kubagawa - Rua José Machado Ribeiro, 54 - Bairro do Limão Cep: 02722-170 - São Paulo, SP - Brasil. E-mail: lívia_kubagawa@hotmail.com.
} 


\section{INTRODUÇÃO}

A próstata, glândula de dimensôes diminutas, localizada na base da bexiga, pode ser sede de dois processos distintos. O primeiro é o crescimento benigno, chamado de hiperplasia, que acomete quase $90 \%$ dos homens após os 40 anos e que produz dificuldade para a eliminação da urina. O segundo é o câncer de próstata, que surge associado ou não ao crescimento benigno e que se manifesta quase sempre depois que os homens completam 50 anos. ${ }^{1}$

O câncer de próstata é o tumor mais freqüente no homem brasileiro, sendo as estimativas do INCA de 25.600 casos novos em 2002. Em 1999, foi responsável por 7.223 óbitos. Fatores genéticos hereditários, alterações em genes somáticos e hábitos alimentares estão relacionados ao desenvolvimento dessa neoplasia. ${ }^{2}$

A prostatectomia radical é o mais antigo e possivelmente o mais eficaz método de tratamento do câncer de próstata localizado. No entanto, essa operação causa muitas complicações, entre as quais a incontinência urinária é a mais aflitiva ${ }^{3}$. Em muitos pacientes, a incontinência melhora em alguns dias, semanas ou meses sem intervenção. Em uma pequena proporção de pacientes, isso não ocorre. ${ }^{4}$

O tratamento da incontinência após a cirurgia depende do seu mecanismo, da sua importância e do tempo pós-cirúrgico. Pacientes com incontinência causada pela hiperatividade da bexiga são bons candidatos para a fisioterapia, e normalmente adquirem a continência normal em um ano. Todavia, pacientes que possuem incontinência de esforço persistente são mais difíceis para tratar. ${ }^{5}$

Para os pacientes que tiveram perda urinária no pósoperatório, exercícios para a musculatura pélvica com biofeedback têm sido sugeridos para melhorar os sintomas e favorecer o retorno do controle urinário ${ }^{6}$. A eletroestimulação também é um método que pode favorecer o sucesso dos exercícios para a musculatura pélvica em pacientes com incontinência após a prostatectomia. $^{7}$

De acordo com Kamben et al apud Kakihara, ${ }^{8}$ é importante assinalar que a reeducação do assoalho pélvico com trabalho da cinesioterapia para fortalecimento do músculo elevador do ânus deve ser considerada como a primeira opção no tratamento da incontinência urinária pós-prostatectomia radical.

Esse trabalho consiste em uma revisão bibliográfica sobre os recursos fisioterapêuticos para o tratamento da incontinência urinária após prostatectomia com o objetivo de discutir a eficácia da fisioterapia na recuperação do controle urinário.

\section{METODOLOGIA}

O levantamento bibliográfico foi realizado com publicações entre os anos de 1997 a 2005 e pela Internet através do site da Bireme para consulta de seus acervos de dados como Lilacs, Medline, PubMed e Cochrane. As palavras-chave utilizadas foram fisioterapia, incontinência urinária, prostatectomia, exercícios para o assoalho pélvico, biofeedback, eletroestimulação e próstata. Todo material adquirido (livros, artigos científicos, teses, dissertaçóes e acervos de audiovisual) foi arquivado e separado pelos diversos tópicos do trabalho. Esse material após lido e analisado foi comparado para avaliação da eficácia ou não dos tratamentos de acordo com os diferentes autores. $\mathrm{O}$ trabalho baseou-se no que foi encontrado na revisão literária realizada e desenvolvido de acordo com as normas da ABNT.

\section{Desenvolvimento}

A fisioterapia é considerada um tratamento adicional para incontinência urinária, e raramente é descrita como forma de tratamento padrão em estudos sobre a incontinência após a prostatectomia. ${ }^{9}$

\section{Exercícios para 0 assoalho pélvico}

Em 1948, Kegel foi o primeiro a preconizar exercícios para a musculatura do assoalho pélvico, para aumentar a resistência uretral e promover o controle urinário. A aplicação dos exercícios de Kegel tem sido expandida a diversas aplicaçóes, mas a sua principal indicação continua sendo para a incontinência secundária à deficiência do esfíncter. O objetivo dessa terapia é a conscientização da existência e da função do assoalho pélvico. Como em qualquer cirurgia, a musculatura geral da região permanece inibida, por isso ela deve ser treinada para recuperar o nível de força normal. ${ }^{10}$

Estudos como os de Chang et al. ${ }^{11}$, Moore, Griffiths e Hughton ${ }^{7}$, VAN Kampen et al. ${ }^{9}$ e Kakihara ${ }^{8}$ verificaram que a cinesioterapia é eficaz quanto à redução dos sintomas urinários, como: a diminuição da perda urinária devido ao aumento da força de contração da musculatura pélvica, aumento do intervalo entre as micções e conseqüentemente diminuição da freqüência urinária, diminuição do grau de incontinência e também maior satisfação dos pacientes 
quanto à qualidade de vida. Parekh et al. ${ }^{10}$ também verificaram que exercícios para o assoalho pélvico no pré-operatório, e reiniciados logo após a retida da sonda vesical acelerariam a recuperação da continência.

\section{Treinamento comportamental}

Um tratamento alternativo e conservador é o treinamento comportamental, usando exercícios para a musculatura pélvica, para ganho de força e resistência do assoalho pélvico. Para realizar a contração correta e isolada desses músculos, o paciente pode aprender a técnica através de alguns métodos: métodos comportamentais, incluindo exercícios sob instruções verbais ou usando biofeedback. Porém, há poucos estudos publicados sobre o papel desses métodos comportamentais na abordagem da incontinência após a prostatectomia ${ }^{3}$. No entanto, o treinamento comportamental é um tratamento inicial apropriado porque é não-invasivo e evita os riscos de efeitos colaterais como os tratamentos médicos e cirúrgicos, além de estar baseado na hipótese de que as respostas da bexiga e o controle do esfíncter são fisiologicamente adquiridos e podem ser reaprendidos ${ }^{6}$.

\section{Biofeedback}

Outro método é o biofeedback que se baseia na transmissão de conhecimentos, para o paciente, a respeito do processo biológico em questão, a incontinência urinária, objetivando um controle voluntário sobre esse processo, a partir dos sintomas e sinais por ele apresentados. ${ }^{12}$

$\mathrm{O}$ biofeedback tem um efeito modulatório sobre o Sistema Nervoso Central (SNC). O treinamento do controle voluntário eficiente da função do assoalho pélvico é capaz de reestabilizar os circuitos neuronais e otimizar a função dos alvos periféricos. Estes mecanismos são controlados pelo SNC. A plasticidade do SNC permite a ação desses mecanismos. ${ }^{13}$

Embora as técnicas de biofeedback possam acelerar o retorno da continência e/ou melhorar o controle urinário após a prostatectomia em alguns pacientes, existe um alto custo com o uso desse método. Críticos do biofeedback, em pacientes prostatectomizados, têm discutido a eficácia desse método e que os custos associados não são justificados. ${ }^{6}$

Teoricamente, o ganho de força dos músculos do assoalho pélvico, seguido do treinamento com biofeedback, associado a exercícios específicos, para esta musculatura, promoverá a realização do mecanismo de fechamento uretral primário. A melhora da conscientização, da força e o treinamento dessa musculatura devem levar a uma contração profilática como um ato motor automático contra eventos de esforço, isso auxilia a diminuírem os episódios de incontinência e a freqüência da troca de absorventes para um retorno mais rápido da continência após a prostatectomia. ${ }^{10}$

Floratos et al. ${ }^{3}$ e Bales et al. ${ }^{6}$, em seus estudos, concluíram que o biofeedback também é eficaz para reduzir sintomas urinários, porém também foi concluído que esse não intensifica os efeitos dos exercícios para a musculatura pélvica quanto à melhora da incontinência e nem quanto ao tempo de recuperação da continência.

\section{Eletroestimulação}

Outra forma de ganho muscular pode ser feita através da estimulação elétrica com o uso de dispositivos cutâneos, endo-anais. Eletrodos colocados no períneo por via percutânea, entre outros, promovem um aumento na resistência esfincteriana e redução na contração detrusora (estimulação dos nervos pudendo e pélvico). ${ }^{12}$

O mecanismo preciso de ação da eletroestimulação em humanos ainda não está comprovado, embora tenha sido demonstrado que o estímulo sensório que segue pelo nervo pudendo pode inibir a atividade do detrusor em homens. Muitos especialistas acreditam que nenhuma forma de estimulação elétrica implantada consiga, através da estimulação dos nervos aferentes pudendo, pelo percurso eferente, causar a contração da musculatura pélvica estriada. Há uma inibição da atividade inapropriada do detrusor, embora o mecanismo aferente ainda não tenha sido esclarecido. Existe um consenso de que a ação da musculatura estriada é capaz de promover a inibição do detrusor neste contexto. Todavia, os dados que suportam esta hipótese permanecem incompletos. ${ }^{14,15}$

A estimulação crônica fortalece a musculatura estriada e a hipertrofia, as fibras de contração rápida e lenta. Acredita-se que a eletroestimulação é uma terapia neuromoduladora a qual afeta os sinais neurais que controlam a incontinência, porém isso é alcançado apenas pela estimulação crônica. ${ }^{16}$

Não há relatos de morbidade significante dessa forma de terapia. Efeitos colaterais que são comuns com tratamento medicamentoso não acompanham este tratamento, porém alguns pacientes relatam algum desconforto ou irritação local. Pacientes com marcapasso (ou outros implantes elétricos) devem considerar métodos alternativos de tratamento, baseados teoricamente nos riscos elétricos. No entanto, não há nenhuma análise econômica dessa terapia. Sabe-se que, nos Estados Unidos, estes estimuladores são muito caros, 
portanto eles não têm muita popularidade entre os especialistas que tratam a incontinência. ${ }^{15}$

Wille et al. ${ }^{16}$ avaliaram os efeitos dos exercícios para a musculatura pélvica, eletroestimulação e biofeedback na incontinência urinária após a prostatectomia radical randomizando 139 pacientes em três grupos que realizariam respectivamente cinesioterapia, cinesioterapia mais biofeedback e cinesioterapia, biofeedback e eletroestimulação. Foi verificado que as diferenças entre os grupos não eram estatisticamente significantes. Portanto conclui-se que os tratamentos com eletroestimulação e biofeedback contribuem para redução dos sintomas urinários, mas não otimizam os efeitos dos exercícios na reabilitação da incontinência.

\section{DISCUSSÃO}

O tratamento da incontinência pós-cirúrgica depende do seu mecanismo, da sua importância e do tempo pósoperatório ${ }^{5,14}$. De acordo com Zermann et al. ${ }^{17}$ a fisioterapia deve ser iniciada um dia após a retirada da sonda vesical para a obtenção da continência o mais rápido possível. Também, é viável iniciar os exercícios antes da retirada da sonda vesical. Além disso, um estudo urodinâmico é essencial para uma avaliação detalhada da incontinência pós-prostatectomia e um exame retal digital é necessário para avaliar o trabalho muscular pélvico. Eles também reportam a importância do estadiamento do tumor, pois o tamanho anatômico da próstata afeta o procedimento cirúrgico durante o qual pode-se causar uma possível lesão da musculatura estriada.

Nenhum estudo citado acima especificou o tipo de incontinência apresentada pelos pacientes. O tempo após a cirurgia, em que se realizou a primeira avaliação e iniciou-se a terapia, foi variado entre os estudos; alguns logo após a retirada da sonda vesical e outros semanas após a cirurgia. A média de idade dos pacientes também foi diferente entre os estudos. Quanto aos critérios de avaliação, apenas Bales et al. $^{6}$ consideraram o estadiamento do tumor como critério de inclusão no estudo, Chang et al. ${ }^{11} \mathrm{e}$ Wille et al. ${ }^{16}$ utilizaram testes urodinâmicos para avaliar a incontinência de forma mais objetiva e não somente os pad tests e questionários sobre sintomas urinários, que são mais subjetivos. Nenhum estudo avaliou a força muscular do assoalho pélvico antes e após a cirurgia. $\mathrm{O}$ período de acompanhamento dos pacientes depois da prostatectomia foi diversificado, e o número de participantes dos estudos, também, variou desde apenas 9 no estudo de Kakihara, ${ }^{8}$ até 139 no estudo de Wille et al. ${ }^{16}$. Por sua vez, Wille et al. ${ }^{16}$ não explicam quais foram e como foram aplicados os exercícios para a musculatura pélvica, e nenhum estudo especificou qual a técnica cirúrgica utilizada. Somente Bales et al. ${ }^{6}$ e Parekh et al. ${ }^{10}$ relataram que foi o mesmo cirurgião o qual fez as prostatectomias do estudo e Floratos et al. ${ }^{3}$ relataram que quatro cirurgiōes realizaram as operações.

\section{CONCLUSÃO}

Verificamos através dessa revisão a importância do tratamento fisioterapêutico (exercícios para o assoalho pélvico, eletroestimulação e biofeedback), na reabilitação da incontinência urinária, após prostatectomia radical, como tratamento do câncer de próstata localizado. Por meio da bibliografia consultada, foi possível notificar a eficácia da fisioterapia quanto aos sintomas urinários, como: a diminuição da perda urinária devido ao aumento da força de contração da musculatura pélvica, aumento do intervalo entre as micções e, conseqüentemente, diminuição da freqüência urinária, diminuição do grau de incontinência e também maior satisfação dos pacientes quanto à qualidade de vida. Além disso, a fisioterapia no pré e pós-operatórios logo após a retirada da sonda vesical mostrou-se mais efetiva do que apenas a fisioterapia tardia semanas após a cirurgia ao reduzir o tempo para a obtenção da continência, sendo seus melhores resultados nos primeiros quatro meses do pós-operatório.

No entanto, não houve diferenças significantes entre os tratamentos com exercícios para o assoalho pélvico, eletroestimulação e biofeedback, ou seja, a hipótese de que a eletroestimulação e o biofeedback otimizariam os efeitos dos exercícios não foi comprovada pelos autores consultados.

Ao realizar esse trabalho, notamos a carência de publicaçôes sobre o tema. Em contra partida, a incidência da incontinência urinária masculina ainda é alta, o que demonstra a necessidade de mais estudos controlados e randomizados para ampliar o conhecimento do tema e dessa forma proporcionar ao paciente a melhor opção de tratamento.

\section{REFERÊNCIAS BIBLIOGRÁFICAS}

01. Srougi M. Câncer de próstata: uma opiniāo médica. Urologia on line [periódico na Internet]. 1998 Out-Dez [citado em Set. 2005]; 2(5):[cerca de 3p.]. Disponível em: http:// www.unifesp.br/dcir/urologia/uroline/ed1098/tela.htm.

02. Leite KRM. A etiopatogenia do câncer de próstata. Urologia on line [periódico na Internet] 2004 Mar [citado em Set. 
2005]; 6(10): [cerca de 3p.]. Disponível em: http:// www.unifesp.br/dcir/urologia/uronline1/art10.htm

03. Floratos DL, Sonke GS, Rapidou CA, Alivizatos GJ, Deliveliotis C, Constantinides CA, et al. Biofeedback vs verbal feedback as learning tools for pelvic muscle exercise in the early management of urinary incontinence after radical prostatectomy. BJU Int. 2002;89(7):714-9.

04. Van Kampen M, De Weerdt W, Van Poppel H, Baert L. Urinary incontinence following transurethral, transvesical and radical prostatectomy: retrospective study of 489 patients. Acta Urol Belg. 1997;65(4):1-7.

05. Peyromaure M, Ravery V, Boccon-Gibod L. The management of stress urionary incontinence after radical prostatectomy. BJU Int. 2002;90(2):155-61.

06. Bales GT, Gerber GS, Minor TX, Mhoon DA, McFarland JM, Kim HL, et al. Effect of preoperative biofeedback / pelvic floor training on continence in men undergoing radical prostatectomy. Urology. 2000;56(4):627-30.

07. Moore KN, Griffiths D, Hughton A. Urinary incontinence after radical prostatectomy: a randomized controlled trial comparing pelvic muscle exercises with or without electrical stimulation. BJU Int. 1999;83(1):57-65.

08. Kakihara CT. Cinesioterapia na redução da incontinência urinária de pacientes pós-prostatectomizados. Fisioter Brasil. 2003; 4(4):265-70.

09. Van Kampen M, De Weerdt W, Van Poppel H, De Ridder $\mathrm{D}$, Feys H, Baert L. Effect of pelvic-floor re-education on duration and degree of incontinence after radical prostatectomy: a randomized controlled trial. Lancet.
2000;355(9198):98-102.

10. Parekh AR, Feng MI, Kirages D, Bremner H, Kaswick J, Aboseif S. The role of pelvic floor exercises on postprostatectomy incontinence. J Urol. 2003;170(1):130-3.

11. Chang PL, Tsai LH, Huang ST, Wang TM, Hsieh $\mathrm{ML}$, Tsui KH. The early effect of pelvic floor muscle exercise after transurethral prostatectomy. J Urol. $1998 ; 160(2): 402-5$.

12. Truzzi JCCI. Tratamento clínico da incontinência urinária masculina. Urologia on line [periódico na Internet]. 1997 Abr-Jun [citado em Set. 2005]; 1(2): [cerca de 3 p.]. Disponível em: http://www.unifesp.br/dcir/urologia/ uronline/ed0397/iumasc.htm

13. Zermann DH, Ishigooka M, Wunderlich $\mathrm{H}$, Reichelt $\mathrm{O}$, Schubert J. A study of pelvic floor function pré and postradical prostatectomy using clinical neurological investigations, urodynamics and eletromyography. Eur Urol. 2000;37:72- 8 .

14. Yalla SV. Managemente of urinary incontinence: progress and innovate strategies [editorial]. J Urol. 1998; 159:1520-2.

15. Brubaker L. Electrical stimulation in overactive bladder. Urology. 2000; 55: 17-23.

16. Wille S, Sobottka A, Heidenreich A, Hofmann R. Pelvic floor exercises, electrical stimulation and biofeedback after radical prostatectomy: results of a prospective randomized trial. J Urol. 2003;170 (2 Pt 1):490-3.

17. Zermann DH, Wunderlich H, Reichelt O, Schubert J. Re: early post-prostatectomy pelvic floor biofeedback [letter]. J Urol. 2000;164 (3 Pt 1):783-4. 